\title{
ENERGETIC PARTICLE PHYSICS STUDIES ON ALCATOR C-MOD
}

\author{
J. A. SNIPES, * N. BASSE, P. BONOLI, C. BOSWELL, and E. EDLUND \\ Massachusetts Institute of Technology, Plasma Science and Fusion Center, Cambridge, Massachusetts 02139
}

A. FASOLI CRPP, Association Euratom-Confederation Suisse, EPFL Lausanne, Switzerland

R. S. GRANETZ, L. LIN, Y. LIN, R. PARKER, M. PORKOLAB, J. SEARS, V. TANG, and S. WUKITCH Plasma Science and Fusion Center, Massachusetts Institute of Technology

Cambridge, Massachusetts 02139

Received April 19, 2005

Accepted for Publication July 30, 2005

Energetic particle physics is studied in Alcator C-Mod in reactor relevant regimes with high density and equilibrated electron and ion temperatures. Stable Alfvén eigenmodes are excited with low-power active magnetohydrodynamic antennas in the absence of a significant energetic particle tail to directly measure the damping rate of the modes. Stable toroidal Alfvén eigenmode (TAE) damping rates between $0.5 \%<\gamma / \omega<4.5 \%$ have been observed in diverted and limited plasmas. Alfvén eigenmodes are destabilized with high-power hydrogen minority ion cyclotron radio frequency $(I C R F)$ heating $\left(P_{\text {ICRF }}<\right.$ $6 \mathrm{MW}$ ) in lower-density plasmas in the current rise and in relatively high-density $\left(\bar{n}_{e}<2.5 \times 10^{20} \mathrm{~m}^{-3}\right) \mathrm{H}$-mode plasmas, which creates an energetic hydrogen ion tail with calculated energies up to $400 \mathrm{keV}$. Low toroidal mode number $(n<4)$ unstable modes are observed in the current rise with magnetic pickup coils at the wall and phase contrast imaging density fluctuation measurements in the core. Observations of energetic particle modes or TAEs that decrease in frequency and mode number with time up to a large sawtooth collapse indicate that fast particles play a role in stabilizing sawteeth. Alfvén eigenmodes can also be used as diagnostics to precisely constrain the q profile and provide a qualitative measure of the fast particle distribution time evolution.

KEYWORDS: Alfvén eigenmodes, Alcator C-Mod, MHD

NOTE: Some figures in this paper are in color only in the electronic version.

\section{INTRODUCTION}

Energetic $\alpha$ particles that will be produced in fusion reactions in future burning plasma experiments such as the International Thermonuclear Experimental Reactor ${ }^{1}$ (ITER) are expected to excite moderate toroidal mode number $n$ Alfvén eigenmodes. ${ }^{2-4}$ If the modes are large enough to transport substantial amounts of hot $\alpha$ particles from the core of the plasma before they give up their energy to the surrounding bulk plasma, they could quench the fusion burn or damage the first wall. ${ }^{5}$ Alcator C-Mod ${ }^{6}$ plays an important role in the study of energetic particle physics because it operates in the same magnetic config-

*E-mail: snipes@psfc.mit.edu uration as proposed next-step devices, at the same density and toroidal field as expected in ITER and in a regime with equilibrated electron and ion temperatures as in future burning plasma experiments. The energetic ions that destabilize AEs in present devices are produced by neutral beam injection or ion cyclotron radio frequency (ICRF) heating. The growth rate of these modes depends on a balance of energetic particle drive ${ }^{7-9}$ with a number of possible damping mechanisms. ${ }^{10-12}$

To separate the damping from the drive, experiments have been performed on the Joint European Torus (JET) with low toroidal mode number, $n \leq 2$, active magnetohydrodynamic (MHD) antennas ${ }^{13-15}$ that excite stable AEs in the plasma at the expected mode frequency, which for toroidal Alfvén eigenmodes (TAEs) is given 
approximately by the center of the gap frequency ${ }^{16} \omega_{\mathrm{TAE}}=$ $v_{A} /(2 q R)$, where $v_{A} \propto B /\left(m_{i} n_{i}\right)^{0.5}$ is the Alfvén velocity. In the approximation used here, $B$ is the magnetic field on axis, $m_{i}$ is the effective ion mass, $n_{i}$ is the ion density, $q$ is the safety factor with the strongest mode coupling, and $R$ is the major radius of the magnetic axis. These stable modes are excited in the absence of a significant energetic particle tail to ensure that the energetic particle drive terms are zero in order to isolate the damping rate of the mode. On Alcator C-Mod, similar experiments were performed with active MHD antennas ${ }^{17,18}$ that can drive stable modes at low power $(\sim 3 \mathrm{~kW})$ with a broad toroidal mode number spectrum $\left(|n|_{\text {fwhm }} \leq 16\right)$, since moderate $n$ are expected to be the most unstable in ITER (Ref. 4). Two arrays of similar moderate- $n$ antennas will soon be installed in JET to better select the excited mode structure. ${ }^{15}$ The dependence of the damping rate on plasma parameters in different machines is then compared with theory to improve the prediction of AE stability limits in future burning plasma experiments.

Unstable Alfvén eigenmodes have been observed during sawtooth stabilization in the current flattop in JT-60U (Ref. 19), JET (Ref. 20), DIII-D (Ref. 21), and TFTR (Ref. 22). These modes indicate the presence of fast particles that are believed to be responsible for stabilizing the sawteeth. ${ }^{23}$ Analysis of these experiments has shown that fast ions are expelled from within the $q=1$ radius because of the AEs. This redistribution of fast ions weakens sawtooth stabilization, leading to the large sawtooth collapse. Similar unstable AEs are observed during sawtooth stabilization with ion cyclotron resonance heating in Alcator C-Mod even with densities up to $\bar{n}_{e} \approx 2.5 \times$ $10^{20} \mathrm{~m}^{-3}$, indicating that a high-energy fast-ion tail is produced with sufficient ICRF heating.

With strong heating in the current rise, modes with rapidly changing frequency that sweep up to the TAE frequency have been observed in a number of tokamaks, ${ }^{24-27}$ known as Alfvén cascades (ACs). According to the theory of Berk et al., ${ }^{28}$ the rapidly changing frequency of these modes indicates that there is a very flat or reversed shear $q$ profile and, for fixed poloidal $(m)$ and toroidal $(n)$ mode numbers, the frequency should change as a function of the minimum $q$ value:

$$
\omega(t)=\left|\frac{m}{q_{\min }(t)}-n\right| \frac{v_{A}}{R_{0}}+\omega_{0} .
$$

The value of the minimum frequency $\omega_{0}$, where ACs are observed is believed to be determined by continuum damping including pressure effects. This simple model fits the JET data very well and allows the ACs to constrain $q_{\min }$. Together with calculations of the AE structure with the MISHKA code ${ }^{29}$ it is then possible to model the evolution of $q_{\min }$ based on the ACs. In this way, ACs in Alcator C-Mod have been used to model the evolution of the $q$ profile in the current rise of strongly ICRF-heated discharges.

\section{DIAGNOSTICS}

The diagnostics for energetic particle physics studies on C-Mod include arrays of fast poloidal field pickup coils, a pair of active MHD antennas, ${ }^{17}$ the phase contrast imaging (PCI) diagnostic, ${ }^{30}$ and a compact neutral particle analyzer. ${ }^{31}$ The magnetic pickup coils are used together with the active MHD antennas to measure stable Alfvén eigenmode resonances in the plasma excited by the antennas to calculate the damping rate of the eigenmodes. The pickup coils and PCI measure edge magnetic and core density fluctuations, respectively. The compact neutral particle analyzer is a new diagnostic that provides a more direct measure of the fast particles that escape the plasma by charge exchange with a diagnostic neutral beam. For very low density $\left(\bar{n}_{e} \sim 6 \times 10^{19} \mathrm{~m}^{-3}\right)$ plasmas, sufficient signal is detected even without the use of a beam.

Figure 1 shows the diagnostics used to measure AEs on C-Mod. The plasma cross section shows the 32 vertical chords of the PCI diagnostic that view through the plasma core, together with some of the magnetic pickup coils on one of two outboard limiters. The PCI diagnostic is sensitive to wave numbers in the major radial direction from $0.5<k_{R}<6.5 \mathrm{~cm}^{-1}$ and the data are sampled at up to $10 \mathrm{MHz}$. The magnetic pickup coils are normally sampled at $2.5 \mathrm{MHz}$ but can also be sampled at up to $10 \mathrm{MHz}$ and can in principle measure toroidal mode numbers up to $n<75$, if the wavelength of the mode is greater than about $2 \mathrm{~cm}$. Figure $1 \mathrm{~b}$ shows that the two active MHD antennas are located between the full limiter on the left and one of the ICRF antennas that is just visible on the right side of the photo. The active MHD antennas each have five turns beneath the boron nitride protection tiles. More details of these diagnostics can be found in Ref. 31 .

\section{STABLE ALFVÉN EIGENMODE EXPERIMENTS}

The initial active MHD experiments in C-Mod demonstrated that it is possible to excite and detect stable resonant AEs with a moderate- $n$ antenna and to measure the damping rate of these modes. For these experiments, only the upper antenna was excited with an amplifier that coupled up to $3 \mathrm{~kW}$ of power at $10 \mathrm{~A}$. The excited perturbations are so small that they do not significantly perturb the plasma, and the resulting AE resonances $\left(\tilde{B}_{\theta}<\right.$ $10^{-6} \mathrm{~T}$ ) can be observed only with the most sensitive fluctuation diagnostics. Toroidal arrays of three magnetic pickup coils mounted beneath the tiles $10 \mathrm{~cm}$ above and below the outboard midplane on each of two limiters were used to measure the AEs in the plasma through software synchronous detection with the antenna current. They can measure fluctuation levels down to about $\tilde{B}_{\theta} \sim$ $10^{-7} \mathrm{~T}$. The data were sampled for 12 signals throughout the discharge at $2.5 \mathrm{MHz}$. 


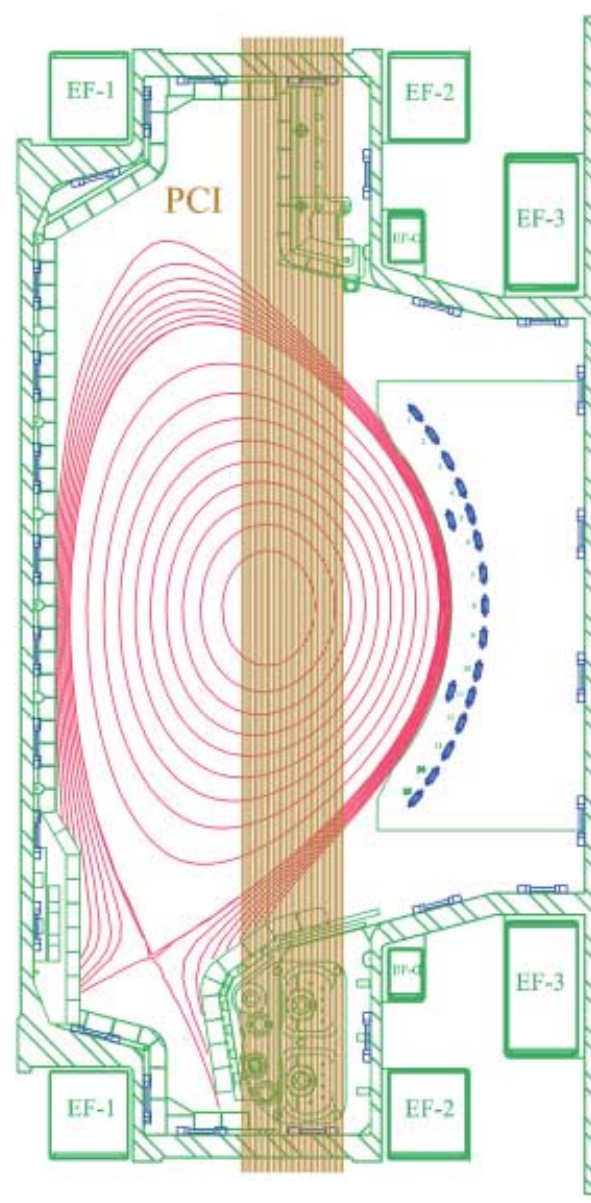

(a)

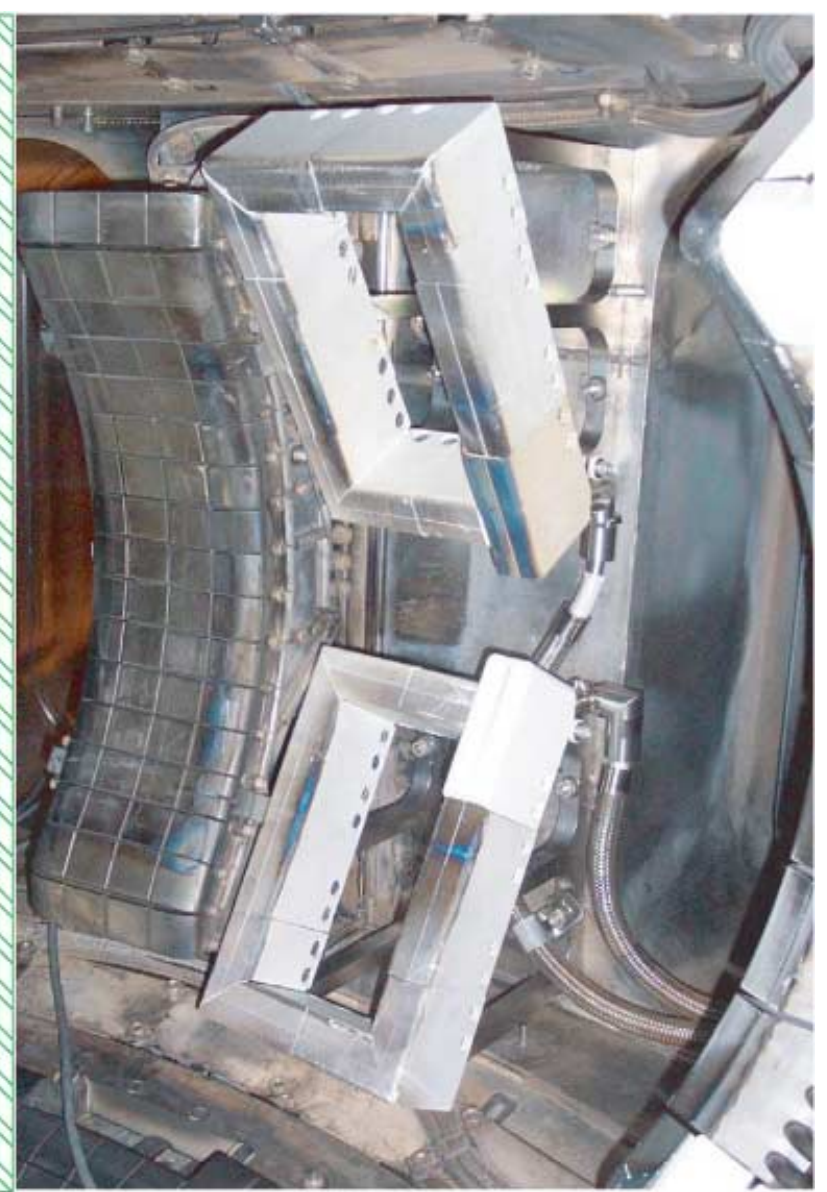

(b)

Fig. 1. (a) Cross section of C-Mod showing the 32 vertical chords of the PCI diagnostic, together with some of the pickup coils (in blue) on one of the outboard limiters, and (b) photo of the two active MHD antennas.

Observable resonances in the pickup coil signals are measured when the excitation frequency of the active MHD system approaches a resonant TAE frequency in the plasma. The mode frequency can lie anywhere within the TAE gap, which has a typical width of $\pm 15 \%$ around the center of the gap frequency. A straightforward way to excite TAE resonances in the plasma is to drive the antenna at constant frequency and then sweep the density or toroidal field so that the center of the TAE gap frequency in the plasma passes through the active MHD frequency. This avoids any problems with matching to the antenna inductance or any resonance in the circuit so that even a rather weak resonance may be observed. Figure 2 shows an example where the toroidal field was ramped down so that the TAE frequency in the plasma passed through the active MHD frequency, and a clear resonance is observed on the Fourier spectrogram of a pickup coil signal. Good matching is obtained with negligible reflected power at constant rms current and voltage by operating at a constant frequency where the antenna inductance has been compensated with capacitors. The measured TAE reso- nances are observed to coincide with the center of the TAE gap frequency calculated at the $q=1.5$ surface using the core line-averaged density, as also observed in JET (Ref. 15). Note that the edge $q$ value changes with toroidal field since the plasma current was held constant. All of these plasmas exhibit small sawteeth indicating that $q_{0} \sim 1$, so a $q=1.5$ surface will be present in the plasma.

To observe multiple resonances in a single discharge and follow the resonance in time, the antenna frequency can be swept up and down through the expected TAE resonant frequency in the plasma. To maintain sufficient matching between the antenna and the amplifier to observe resonances, the frequency could be swept by at most $\pm 50 \mathrm{kHz}$. With a sufficiently flexible amplifier and matching circuit, it may be possible to feedback on the measured stable mode resonances together with current and pressure profile control heating systems to control the AE perturbations, before they become unstable, and help to control the fusion burn in a future burning plasma experiment. 

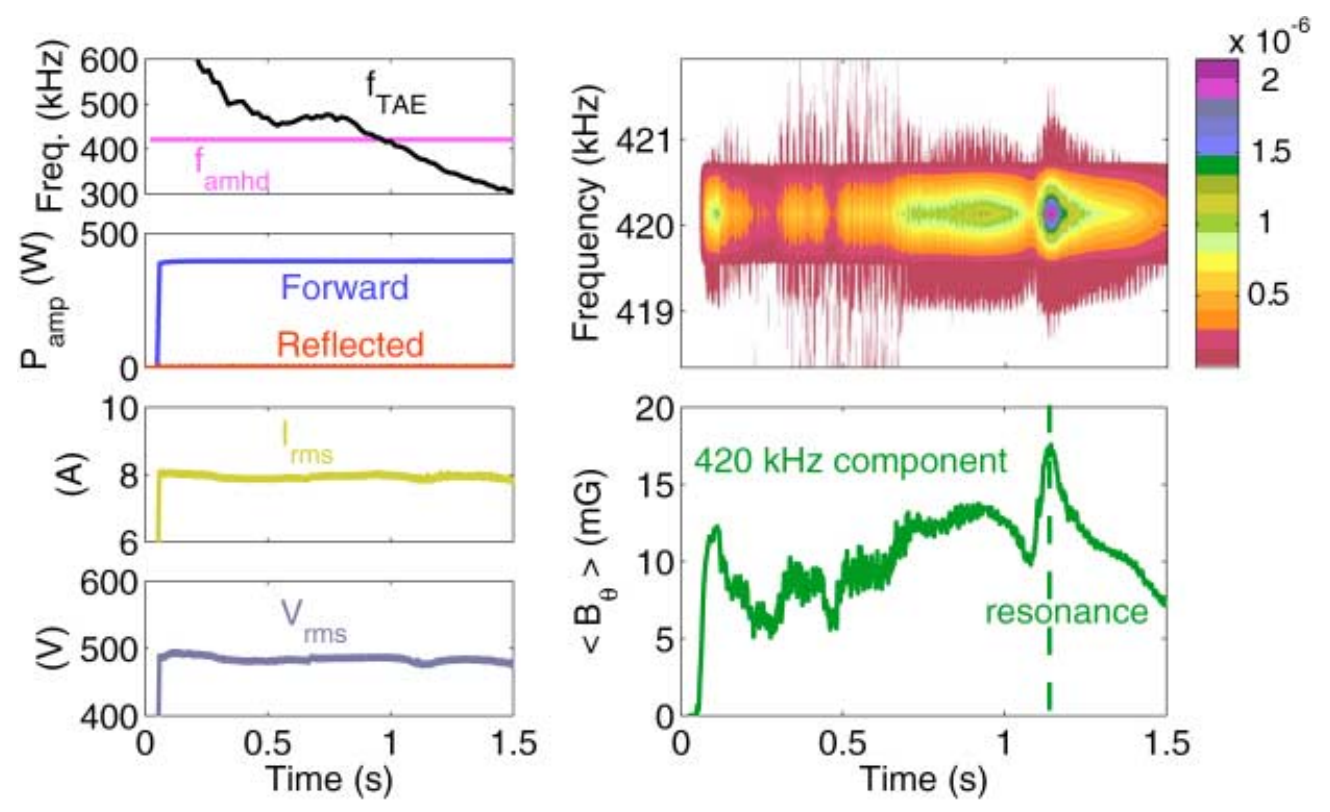

Fig. 2. Fourier analysis spectrogram of a typical TAE resonance in C-Mod with the active MHD antenna excited at a constant frequency of $420 \mathrm{kHz}$ and the plasma TAE frequency swept across the antenna frequency by ramping the toroidal field from 6.5 to $4 \mathrm{~T}$. A resonance is clearly visible in both the spectrogram and the average poloidal field fluctuation versus time as the plasma center of the TAE gap frequency crosses the active MHD frequency. The rms current and voltage across the antenna are shown, together with the forward and nearly zero reflected power.

\section{III.A. Software Synchronous Detection}

Because the observed mode resonances have small amplitude $\left(\tilde{B}_{\theta} \sim 10^{-6} \mathrm{~T}\right)$, a synchronous detection technique is employed using data from multiple pickup coils to more clearly identify the mode resonances above the background noise level. Rather than using hardware synchronous detection circuits as on JET (Ref. 32), the data were fast sampled $(2.5 \mathrm{MHz})$ and synchronous detection was performed with software to allow flexibility in the analysis of the data.

The pickup coil data are first narrow bandpass filtered within $5 \mathrm{kHz}$ of the excitation frequency and then mixed with a reference provided by the antenna current and a 90-deg phase-shifted reference to provide in-phase and quadrature components, which are then low-pass filtered to $1000 \mathrm{~Hz}$ to distinguish the slowly varying amplitude and phase of the resonance. The ratio of the synchronously detected pickup coil signal to the antenna current then gives the complex transfer function. By comparing toroidally separated coils, the phase of the transfer function can be used to determine the toroidal mode number. The normalized damping rate of the mode is then given by the fitted width of the resonance, $\gamma / \omega_{0}$. With the present signal-to-noise ratio, the minimum damping rate that has been measured has $\gamma / \omega_{0}$ just under $0.2 \%$ with a typical error bar of $\pm 0.2 \%$. A clear resonance in the transfer function should sweep out a circle in the complex plane as the plasma parameters are changed in time to change the TAE frequency in the plasma or as the active frequency is swept through the plasma TAE frequency.

Up to now, real-time measurements of the damping rate have not been made on C-Mod, but it may be possible to do the synchronous detection calculation in real time at least for one coil to be able to track AE resonances in real time throughout the discharge. Streamlining the essential synchronous detection algorithm will be required to make the calculation on a dedicated personal computer within 10 to $20 \mathrm{~ms}$ to be able to follow rapid changes in the relevant plasma parameters. This would allow the damping rate of a particular mode to be followed in time and provide a means for feedback control.

\section{III.B. Moderate-n AE Damping Rate Measurements}

Given the broad toroidal mode spectrum of the active MHD antenna $(|n|<16$ full-width at half-maximum), it is not clear what determines with which toroidal mode number the plasma responds. By comparing the phase of the residues of the transfer functions of toroidally separated pickup coils, the toroidal mode numbers of the resonant modes were found to be in the range $3 \times n \leq 14$, which are in the range of mode numbers expected to be unstable in ITER (Ref. 4). The damping rate for ohmic inner wall-limited plasmas was found to be in the range $1 \%<|\gamma / \omega|<3 \%$, which is in the same range as for JET plasmas. Figure 3 shows an example of resonances 

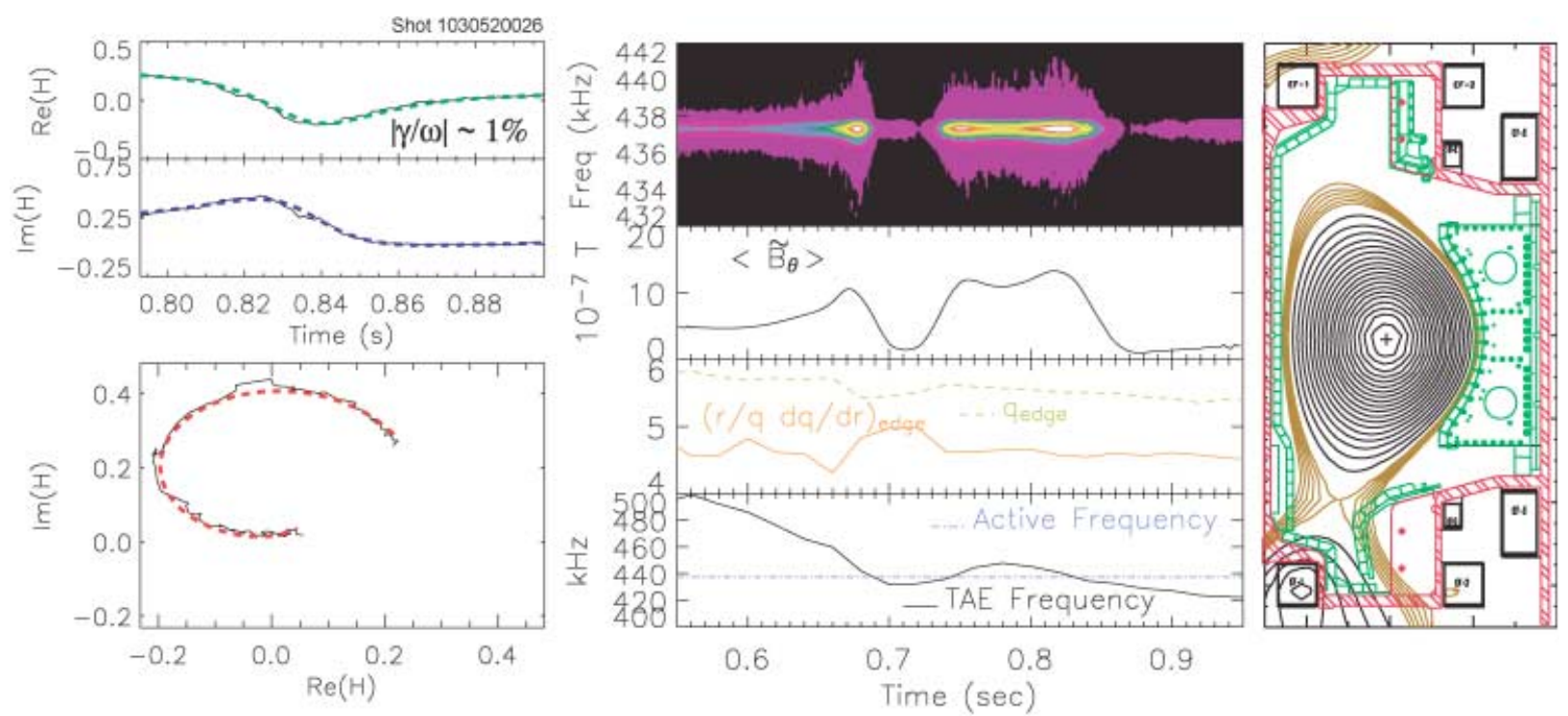

Fig. 3. Three stable TAE resonances observed in a diverted plasma as the plasma TAE frequency passes through the constant active MHD frequency three times. The fit of the transfer function for the third resonance is shown on the left of the figure in the complex plane. The damping rate was found to be particularly low compared to inner wall-limited plasmas with $|\gamma / \omega| \sim 1 \%$.

obtained in a diverted plasma, where the damping rate was found to be $1 \%$, which is lower than the damping rates measured for low- $n$ modes in diverted JET plasmas with high edge shear, which exceed 5 to $10 \%$. The plasma shape and magnetic configuration are shown on the right of the figure. The difference in the measured TAE damping rates between inner wall-limited and diverted ohmic plasmas with ramping toroidal field is shown for a number of discharges during the same run day in Fig. 4. For small outer gaps $<1 \mathrm{~cm}$ in limited plasmas, resonances were not observed. The damping rate of limited plasmas increased with increasing outer gap between 1 and $2.5 \mathrm{~cm}$. For diverted plasmas, on the other hand, only when the outer gap was $<1 \mathrm{~cm}$ were resonances observed, and they had very low damping rates, $0.4 \%<|\gamma / \omega|<1.4 \%$, that appear to decrease with decreasing outer gap. Note that the toroidal mode number is not the same for each of these discharges since with a single antenna it is not possible to select a specific mode number. So, there could also be a dependence on mode number that may mask the true dependence of the damping rate on outer gap. For outer gaps larger than about $1 \mathrm{~cm}$, resonances were no longer observed in diverted plasmas. The lack of observed resonances under these conditions may be due to reduced coupling to the mode or exceedingly high damping rates. Note that plasma rotation measurements were not available for this series of discharges but are expected to be less than $5 \mathrm{kHz}$ in the electron direction based on similar ohmic plasmas.

To further check the dependence of the TAE damping rate on edge shear, an experiment was performed for a number of inner wall-limited ohmic discharges with different elongations. The plasma parameters were $I_{p}=$ $0.7 \mathrm{MA}, 4.7 \mathrm{~T}<B_{T}<5.6 \mathrm{~T}, \bar{n}_{e}=2$ to $2.5 \times 10^{20} \mathrm{~m}^{-3}$, $3.1<q_{95}<4.5$, and $370<f_{\mathrm{TAE}}(q=1.5)<470 \mathrm{kHz}$. The outer gap varied only over a small range from 1.2 to

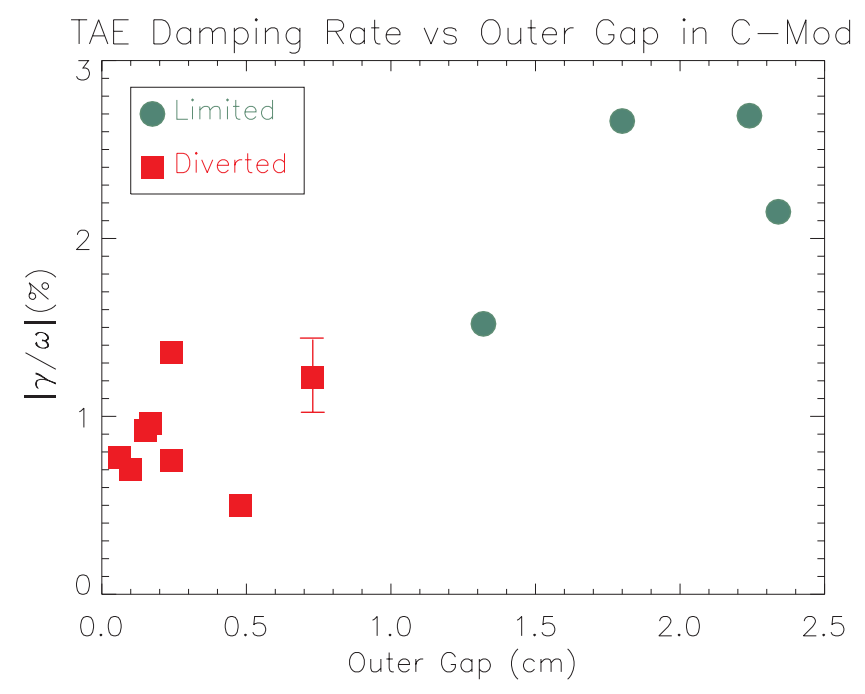

Fig. 4. Stable TAE damping rate as a function of outer gap for both inner wall-limited and diverted plasmas in C-Mod. For outer gaps $<1 \mathrm{~cm}$, resonances were not observed in limited plasmas, whereas for outer gaps $>1 \mathrm{~cm}$, resonances were not observed in diverted plasmas. A typical error bar is also shown. 
$1.7 \mathrm{~cm}$, which should not affect the growth rate substantially. Figure 5 shows the measured damping rates for each discharge as a function of elongation and edge shear calculated at the $95 \%$ flux surface. In contrast to the results on JET for low- $n$ modes, ${ }^{14}$ no clear dependence on edge shear is found for these moderate- $n$ modes, and the damping rate appears to decrease with increasing elongation. Note that with only a single antenna, no selection can be made for the excited mode number. So, it is possible that a dependence of the damping rate on mode number could mask a dependence on edge shear.

The initial active MHD experiments exciting only one antenna on C-Mod have demonstrated that stable TAE resonances can be excited and observed in both inner wall-limited and diverted plasmas in the range of densities and toroidal fields expected in ITER. The resonances were excited at a constant active MHD antenna frequency for ramping toroidal field at various flattop densities. The toroidal field at resonance scaled as the
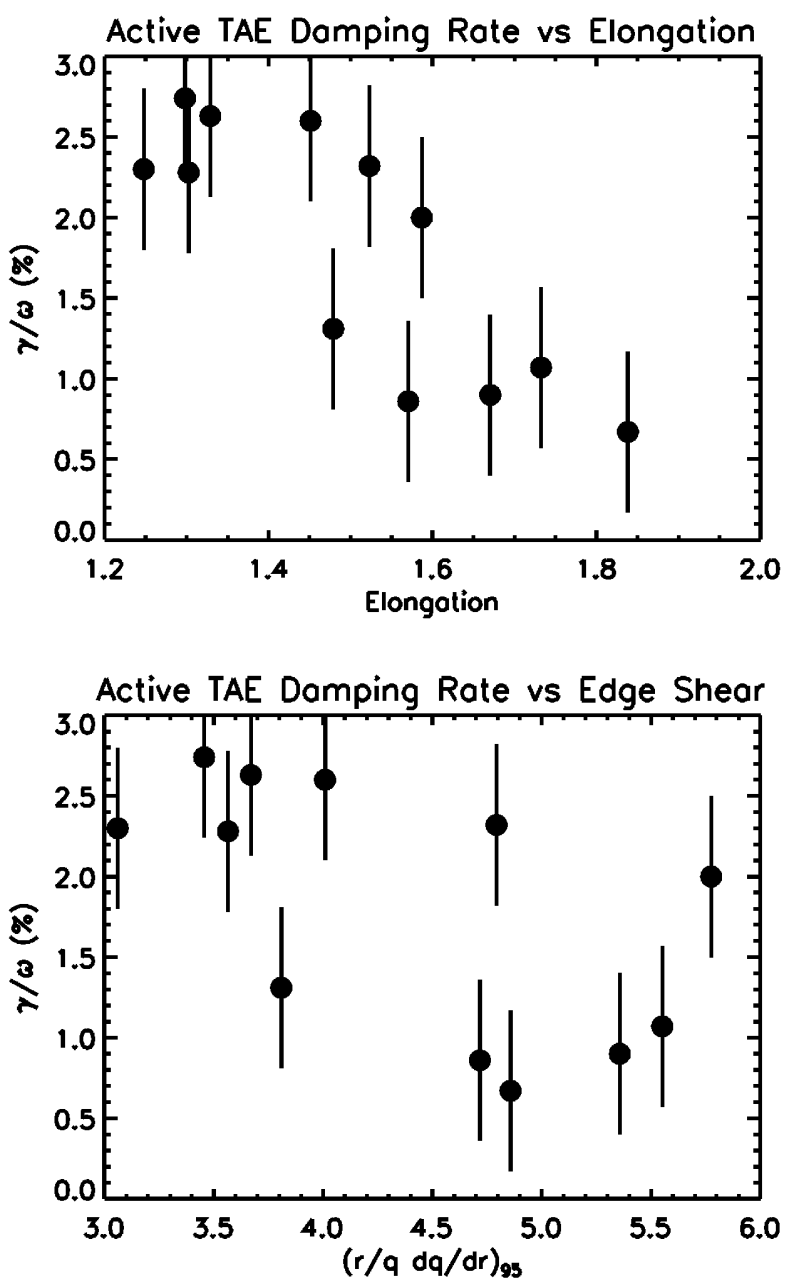

Fig. 5. The measured TAE damping rate as a function of plasma elongation and magnetic shear at the $95 \%$ flux surface for a range of moderate- $n$ modes, $4 \leq n<14$. square root of the line-averaged density as expected for AEs. The resonances are consistent with a center of the TAE gap frequency corresponding to a $q$ value between 1.3 and 1.5 , though core toroidal rotation measurements and mode numbers need to be taken into account to correct for Doppler shifts in the frequency.

The software synchronous detection routine provides good fits to the stable TAE resonances on multiple pickup coil signals. The fitted transfer function in the complex plane yields damping rates between $1.4 \%<$ $|\gamma / \omega|<4 \%$ for inner wall-limited plasmas in agreement with JET results. For diverted plasmas, on the other hand, the resonances were observed only when there were particularly small outer gaps of $<1 \mathrm{~cm}$. In these diverted cases, the damping rates were in the range $0.4 \%<$ $|\gamma / \omega|<1.4 \%$. The apparent discrepancies between the JET low- $n$ damping results and the C-Mod moderate- $n$ damping results could be due to a difference in the behavior of moderate- $n$ modes with respect to low- $n$ modes. However, since a single- $n$ mode has not been tracked, at least some of the discrepancies could also be due to differences in damping rates for different $n$ modes. More experimental results are required to be able to check the dependence of edge shear for a given $n$ mode.

The active MHD amplifier and power supply on C-Mod are presently being upgraded to excite both antennas with up to $20 \mathrm{~A}$ of current simultaneously. ${ }^{33}$ The matching circuit is being designed to automatically switch capacitors to attempt to maintain matching to the antenna as the frequency sweeps across a broad range. A new digital control computer for C-Mod plasma operation will provide a real-time signal to sweep about the TAE frequency as it changes with plasma conditions. These improvements will allow more systematic studies of the damping rate of moderate- $n$ modes as a function of plasma conditions to better refine theoretical expectations for next-step devices.

\section{UNSTABLE AEs DURING THE CURRENT FLATTOP}

Unstable AEs are observed in C-Mod during the current flattop in the presence of sawteeth even at electron densities up to $\bar{n}_{e}=2 \times 10^{20} \mathrm{~m}^{-3}$ when there is sufficient ICRF heating. Mode frequencies corresponding to the TAE and ellipticity-induced AE (EAE) gaps associated with $q=1$ are observed with either constant frequency across a number of sawteeth or with decreasing frequency as $q$ evolves during sawtooth stabilization up to the sawtooth collapse. Note that core plasma rotation frequencies in ICRF-heated plasmas may be as high as $20 \mathrm{kHz}$, so for high toroidal mode numbers, the Doppler shift of the frequency may be significant.

\section{IV.A. Unstable AEs During Enhanced $D_{\alpha}$ H-Mode}

In steady-state enhanced $\mathrm{D}_{\alpha}$ (EDA) H-mode ${ }^{34}$ highfrequency modes are observed with nearly constant 
frequency, such as the $\sim 600-\mathrm{kHz}$ mode shown in Fig. 6 that persists for about $12 \mathrm{~ms}$ after the $\mathrm{rf}$ is switched off. The 500-kHz beat wave between the two ICRF antennas that are driven at 80.0 and $80.5 \mathrm{MHz}$ is also clearly visible, together with several other bursting modes at nearly 150,300 , and $450 \mathrm{kHz}$. The persistence of the highfrequency mode after the rf switches off together with the fact that the frequency is near the center of the TAE gap for $q=1$ suggest that the mode may be fast particle driven. Under these conditions $\left(\bar{n}_{e}=2.5 \times 10^{20} \mathrm{~m}^{-3}\right.$, $\left.T_{e}(0)=2.5 \mathrm{keV}\right)$, the slowing-down time of a $40-\mathrm{keV}$ fast $\mathrm{H}$ ion is about $12 \mathrm{~ms}$. In cases with particularly large sawteeth, the mode frequency is modulated by the sawteeth by about 3 to $5 \%$, with the frequency increasing sharply just after the sawtooth collapse then slowly decreasing during the rise of the next sawtooth (Fig. 7). Such a change in mode frequency with the sawtooth would be consistent with a TAE mode within the $q=1$ surface if the core density fell by 6 to $10 \%$ at the sawtooth collapse. While there are no local density measurements on C-Mod with time resolution high enough to determine the change in density due to the sawteeth, the required change in density is within the error on the measured change in the inverted measurements from the visible bremsstrahlung array. Another possibility is that the flattening of the pressure gradient due to the sawtooth increases the mode frequency through a neoclassical change in the plasma rotation. These modes were also observed in PCI density fluctuation signals, though the frequency was aliased due to the $1-\mathrm{MHz}$ sampling rate of those data. The PCI measurements indicate that the modes have a major radial wave number $k_{R} \approx 1.3 \pm 0.4 \mathrm{~cm}^{-1}$. Although the PCI chords pass through the core of the plasma, they also pass through the plasma edge, so the radial

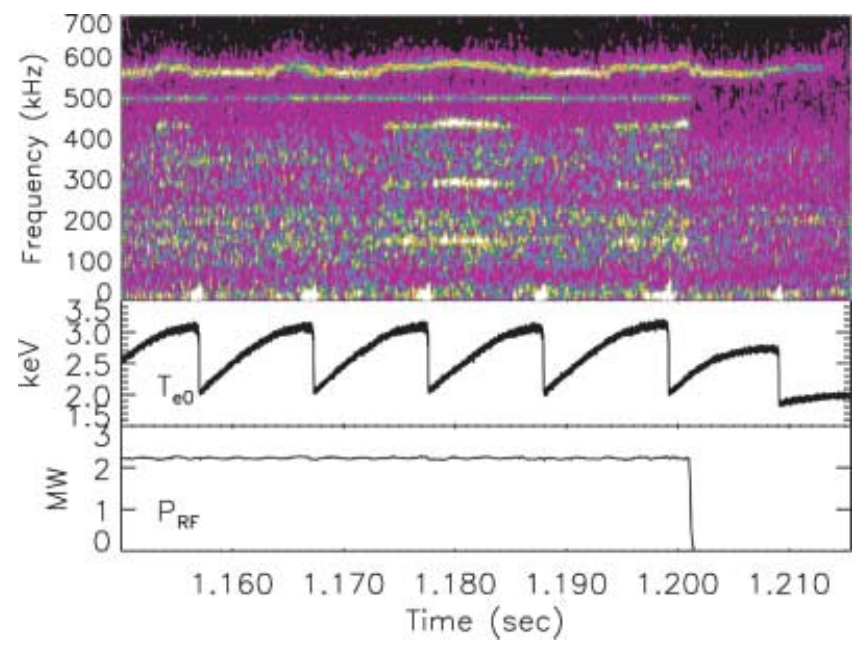

Fig. 6. Fourier spectrogram of a pickup coil signal showing a high-frequency mode near $600 \mathrm{kHz}$ that persists after the ICRF switches off at $1.2 \mathrm{~s}$, together with the central electron temperature and the ICRF power.

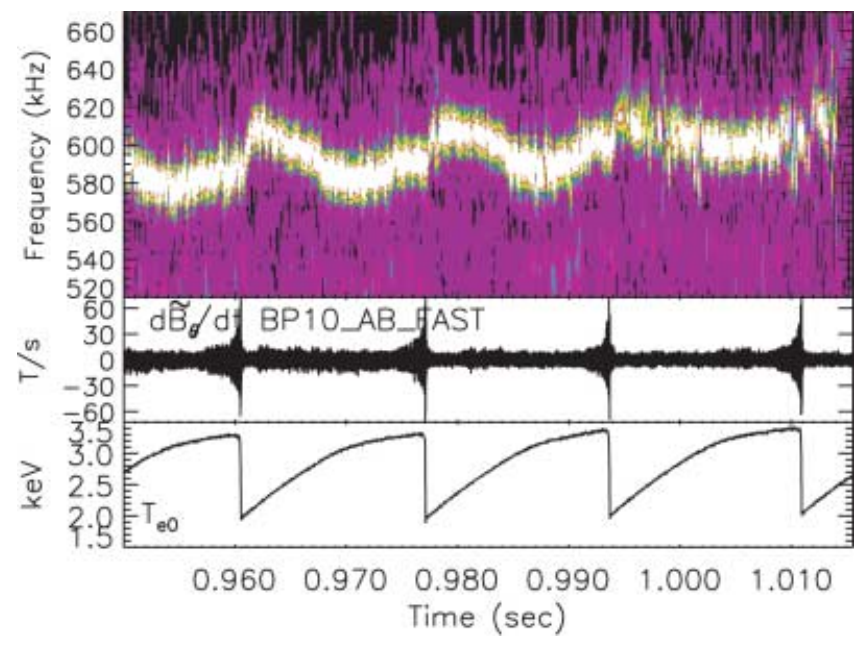

Fig. 7. Fourier spectrogram of a magnetic pickup coil signal showing frequency modulation of a high-frequency mode due to large sawtooth oscillations during an EDA $\mathrm{H}$-mode.

localization of the mode cannot be determined from these data.

As further evidence that these high-frequency modes during EDA H-mode may be fast particle driven, the hydrogen concentration $\left[n_{\mathrm{H}} /\left(n_{\mathrm{H}}+n_{\mathrm{D}}\right)\right]$ was scanned shot to shot from a few percent to more than $25 \%$. Highfrequency magnetic fluctuations similar to those in Fig. 6 were observed only for hydrogen concentrations below about $13 \%$. Only lower-amplitude incoherent fluctuations were observed at higher hydrogen concentrations (Fig. 8). Note that the high-frequency coherent mode amplitudes in the EDA H-mode discharges are about an order of magnitude smaller than those in low shear current rise cases to be discussed in Sec. V. The ICRF power and the electron density were held roughly constant for this series of discharges. At the high hydrogen concentrations, the ICRF heating is expected to go from hydrogen minority heating, which may create a fast-ion tail, to mode conversion heating, which predominantly heats electrons directly and should not generate a fast-ion tail. ${ }^{35}$ So, these data suggest that the high-frequency modes could be driven by a fast-ion tail that goes away at high hydrogen concentration.

These high-frequency modes have typical toroidal mode numbers $n=5$ to 7 and appear to rotate in the electron diamagnetic drift direction. Plasma toroidal rotation, measured by Doppler shift of argon ions in the core, is in the ion diamagnetic drift direction with values of 5 to $25 \mathrm{kHz}$. In some cases, several simultaneous highfrequency modes are observed with increasing frequency and increasing $n$ numbers, such as $n=5(473 \mathrm{kHz}), 8$ $(546 \mathrm{kHz})$, and $11(620 \mathrm{kHz})$, all rotating in the electron diamagnetic drift direction. These data suggest that the multiple- $n$ modes are Doppler shifted by a local plasma 


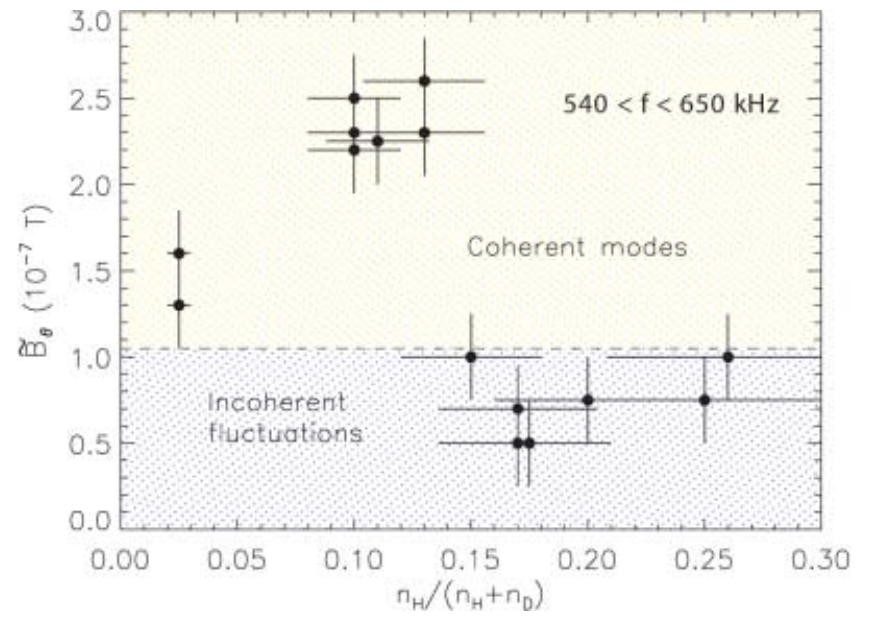

Fig. 8. Magnetic fluctuation amplitude in the frequency interval from 540 to $650 \mathrm{kHz}$ for a series of ICRF-heated EDA H-mode discharges as a function of hydrogen concentration, showing that coherent high-frequency modes are present only when the hydrogen concentration is less than $13 \%$.

rotation of about $25 \mathrm{kHz}$. But since the core plasma rotation is in the direction opposite to the mode rotation, the $n$ numbers should decrease with increasing frequency if the modes were core localized modes. The apparent electron rotation direction of these modes cannot easily be explained if they are core localized fast-particledriven modes. TAE modes would be expected to rotate in the ion diamagnetic drift direction, for a centrally peaked fast-ion distribution. The ICRF resonance in these discharges is generally 1 to $1.5 \mathrm{~cm}$ inside the magnetic axis. This does not seem to be sufficient to obtain a hollow fast-ion pressure profile, which could change the direction of rotation of a TAE mode, as found on JET to explain some cases of TAE modes that rotate in the electron direction. ${ }^{36,37}$

In the absence of another explanation for core TAEs that rotate in the electron diamagnetic drift direction, these data suggest that the high-frequency modes in EDA H-mode may be drift modes that come from the steep gradient edge region, where the rotation is believed to be in the electron diamagnetic drift direction based on lowerfrequency $n=1$ magnetic fluctuation measurements. ${ }^{38}$ ASDEX has reported edge TAE modes in ohmic plasmas that also rotate in the electron diamagnetic drift direction. ${ }^{39}$ In Alcator C-Mod, these modes are observed only in ICRF-heated H-mode and not ohmic $\mathrm{H}$-mode, but it is not clear from these data whether they are resonant in the core or edge region since some data support a core interpretation while others support an edge interpretation. Perhaps the upgraded PCI diagnostic will be able to distinguish between an edge or core localization of these modes in the future.

\section{IV.B. Unstable AEs During Sawtooth Stabilization}

Figure 9 shows an example of sawtooth stabilization with ICRF heating during a double-null discharge. The plasma has a brief $\mathrm{H}$-mode from 0.76 to $0.784 \mathrm{~s}$, where the central line-averaged density rises from 1.6 to nearly $2 \times 10^{20} \mathrm{~m}^{-3}$. The resulting drop in the TAE frequency is evident in the calculated center of the gap frequency for $q=1$ just before the sawtooth collapse that ends the H-mode. A Fourier spectrogram of the AEs and a toroidal mode analysis show that the modes begin with relatively high $|n|=8$ to 10 at about $750 \mathrm{kHz}$, then coalesce as the frequency drops to $650 \mathrm{kHz}$ with increasing density in the H-mode, and the mode numbers also drop to $|n|=4$ to 5 just before the sawtooth collapse. Note that since the plasma current is negative (clockwise viewed from above),
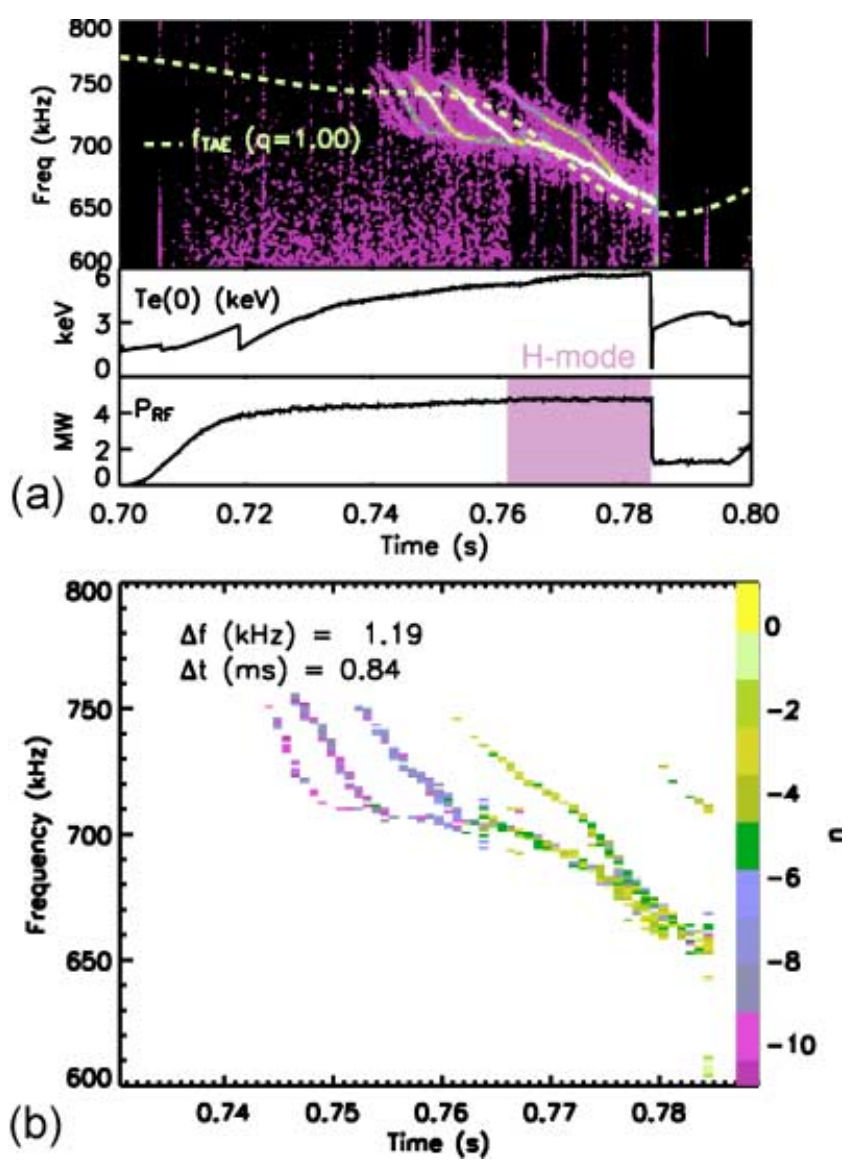

Fig. 9. (a) Fourier spectrogram of an edge poloidal field pickup coil signal showing multiple AEs during sawtooth stabilization, together with the electron temperature measured from electron cyclotron emission and the ICRF power. The plasma is in H-mode during the shaded time. (b) Toroidal mode spectrum showing that the $n$ number begins at $|n|=10$ and decreases to $|n|=4$ just before the sawtooth collapse. Negative $n$ indicates the ion diamagnetic drift direction. The frequency and time resolution of the Fourier transform are also indicated. 
these modes with negative $n$ numbers rotate in the ion diamagnetic drift direction. The coalescing of the modes to a single frequency suggests that the AE gap is closing during the time evolution of the sawtooth or that they reach the bottom of the gap. ${ }^{40}$ One would expect that the previous sawtooth collapse flattens the core $q$ profile, producing a relatively broad TAE gap. Then, as the sawtooth heats up, the current density profile should peak with peaking temperature, which would tend to close the TAE gap until the large sawtooth collapse.

Attempts were made to model the modes with the Nova-K code ${ }^{41}$ at $t=0.74$ and $t=0.78 \mathrm{~s}$ (Ref. 18). Eigenmodes were found in the same range of mode numbers and frequencies as in the experiment. The radial structure at $t=0.74 \mathrm{~s}$ was that expected for TAEs with two dominant harmonics $m$ and $m+1$ peaked at $r / a=$ 0.4 , while the radial structure at $t=0.78 \mathrm{~s}$ had multiple poloidal harmonics between $0.4<r / a<0.9$. The trapped particle total growth rates were found to be stable because of relatively large radiative damping even for flat shear $q$ profiles out to $r / a=0.4$, varying $q_{0}$ from 1 down to 0.8 and varying the assumed fast-ion tail energy from 15 to $700 \mathrm{keV}$. The TRANSP-calculated fast-ion tail energy peaked at about $450 \mathrm{keV}$ for these conditions. This may indicate that these are energetic particle modes ${ }^{42}$ that are not included in the Nova-K code or corelocalized TAEs (Ref. 43) with a different $q$ profile.

The presence of such fast-particle-driven modes during flattop conditions at relatively high density indicates that there is sufficient ICRF heating to drive a significant fast-ion tail. Correlations of such modes with sawtooth stabilization are another indication that a sufficient fastion tail is present to stabilize the sawteeth. Similar modes during EDA H-mode also suggest that they are driven by energetic ions, but the electron diamagnetic drift direction of rotation cannot easily be explained for core modes. This may indicate the need for improved theory to explain these modes.

\section{UNSTABLE ALFVÉN EIGENMODES IN THE CURRENT RISE}

With $\mathrm{H}$ minority ICRF heating in the current and density rise on C-Mod, an energetic $\mathrm{H}$ ion tail is driven that excites rapidly increasing frequency ACs when the minimum in the $q$ profile is substantially greater than unity and has very flat or perhaps slightly reversed shear, which cannot be distinguished within the errors of the calculations. Although not recognized as ACs at the time, these modes were the first AEs observed on C-Mod in the initial current rise ICRF heating experiments ${ }^{26}$ performed in 1996. Figure 10 shows an example of one of the first AEs measured in the current rise. The frequency increases linearly from 150 to $450 \mathrm{kHz}$ in less than $0.015 \mathrm{~s}$, which agrees, within the errors of the calculations, with

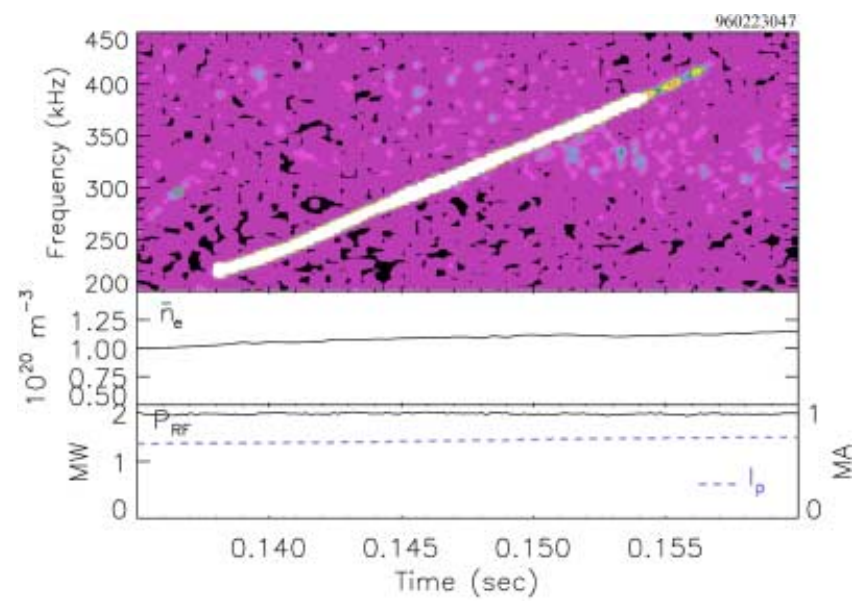

Fig. 10. Fourier spectrogram of magnetic fluctuations during the current rise, showing a narrowband coherent mode with linearly increasing frequency. The line-averaged density, ICRF power, and plasma current are also shown. The frequency evolution can now be explained as an AC.

the frequency evolution expected for an AC. More recent experiments with more than twice as much ICRF power and the addition of the PCI diagnostic have improved our understanding of these modes and the conditions that lead to them. There is no evidence for increased particle or energy transport due to these modes, though fast particle redistributions or losses may not be easily detected with the existing diagnostics. A new compact neutral particle analyzer has recently been installed to attempt to improve measurements of the energetic particle distribution.

\section{V.A. Core and Edge AC Measurements}

In addition to the magnetic pickup coils on the outboard limiters that measure the more radially extended low- $n$ Alfvén eigenmodes, the PCI diagnostic measures the density fluctuations of the AEs along 32 vertical chords passing through the plasma core. Figure 11 shows a comparison of ACs measured with magnetic pickup coils and with PCI. The slopes of the ACs are much higher on the PCI measurements than on the magnetic measurements. According to theory, the slopes are proportional to the $n$ number, implying that the PCI measurements through the core are more sensitive to higher mode numbers than the edge magnetic measurements. Note, however, that the ratios of the slopes of multiple modes within a given cascade do not always remain constant in time throughout the rise of the cascade frequency, which makes it difficult to determine the toroidal mode numbers from the slopes alone. This indicates that either the mode numbers are changing during the frequency rise of the cascade or the expression given by Eq. (1) for the cascade frequency does not fully describe such data. 


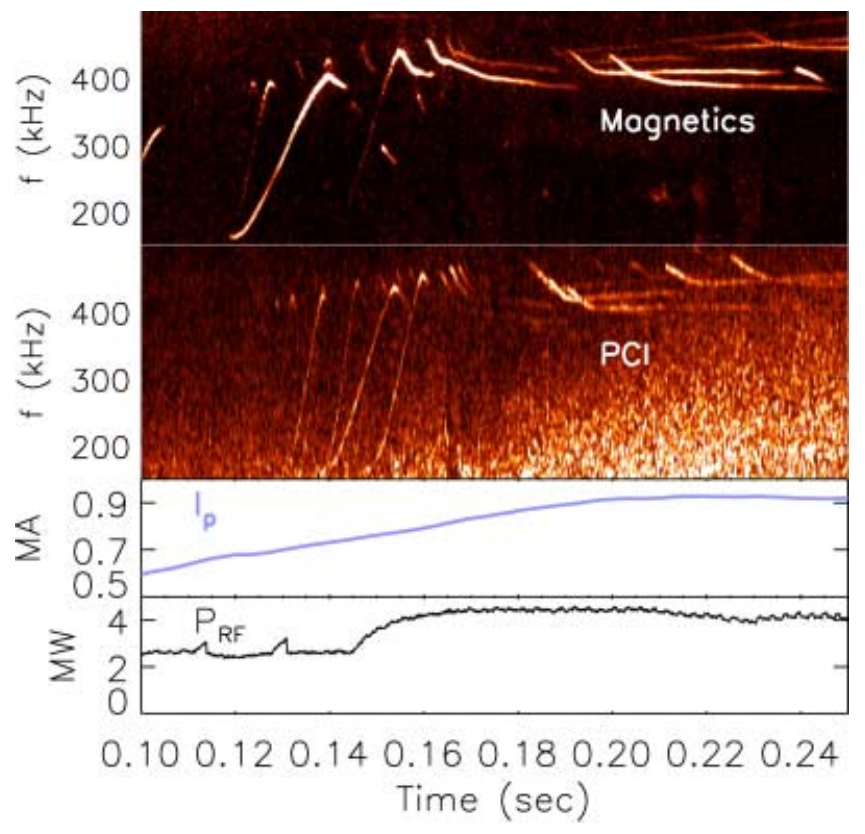

Fig. 11. Fourier spectrograms of an edge poloidal field pickup coil signal and a core PCI signal, showing ACs with steeper slopes in the core than at the edge.

The phase differences between toroidally separated pickup coils during the ACs indicate that the dominant modes are low $n$, but despite detailed in-vessel calibrations of the frequency dependence of the phase differences between the coils, the $n$ numbers appear to change during the upward sweep in frequency for some cascades. This is in contrast to the constant $n$ observed in JET and JT-60U for each frequency sweeping mode. In the case of Fig. 11, the dominant cascades in the magnetic spectrum are $n=1$ to 3 , while the slopes in the PCI data indicate toroidal mode numbers from $n=2$ to 6 . The radial eigenfunctions of the long-wavelength modes extend out to the wall, while those of the shorter-wavelength modes fall off more rapidly with radius and are not large enough to be observed at the wall. Similar differences between edge magnetic and core interferometer cascade measurements have been found recently ${ }^{44}$ on JET. The PCI and magnetic measurements complement each other in that the lowest- $n$ modes are often not visible on the PCI because the sensitivity of the instrument falls off for long-wavelength modes with small $k_{R}$ (Ref. 45 ).

\section{V.B. Modeling the Evolution of the Safety Factor Profile}

Given the $n$ numbers from the slopes of the ACs and the frequency evolution of the modes, the MISHKA code was used to model the evolution of the $q$ profile. Figure 12 shows the calculated evolution of the minimum in $q$ from the measured frequency evolution of the modes in Fig. 11, together with the modeled $q$ profile at the start of
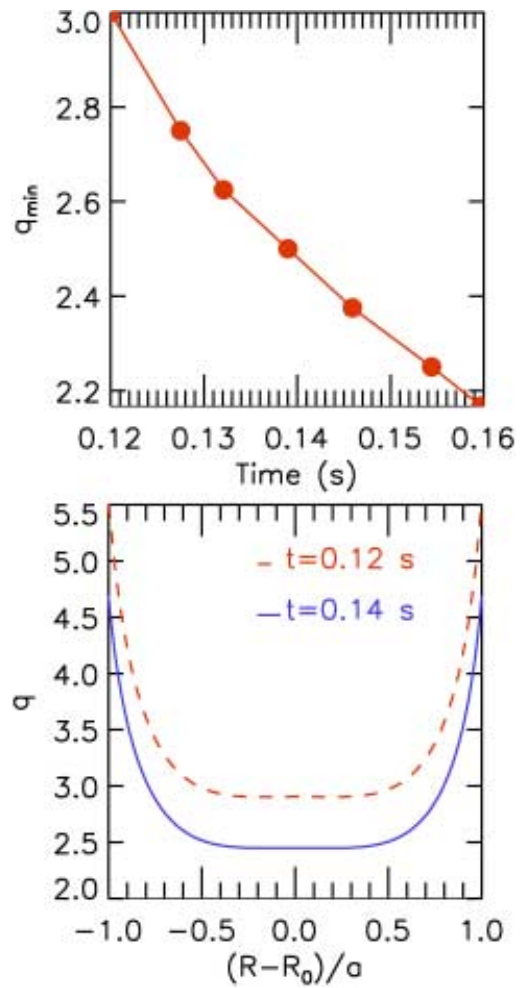

Fig. 12. MISHKA modeling of the evolution of the minimum $q$ value based on the frequency evolution of the ACs of Fig. 5 together with the modeled $q$ profile at $t=$ 0.12 and $0.14 \mathrm{~s}$.

the cascades. The start of an AC with multiple modes like that at $0.12 \mathrm{~s}$ indicates that $q_{\min }$ is passing through an integer value, and then the appearance of another $n=2$ mode at about $0.14 \mathrm{~s}$ indicates that $q_{\mathrm{min}}$ is passing through the next half-integer value. The calculated $q$ profile had very slightly reversed shear that was nearly flat at $q_{\min }$ out to $r / a \approx 0.4$ then rose to $q_{95} \approx 5$.

During the upward frequency sweep of each mode, the calculated mode structure has a core-localized single dominant $m$ characteristic of an AC. The continuum also has a wide gap, which allows the mode frequency to start well below the TAE frequency without being strongly damped by the continuum. Just after the frequency peaks and begins to decrease, the mode structure evolves into a radially broad TAE structure with comparable amplitudes of two poloidal harmonics $m$ and $m+1$. The continuum gap then narrows around the TAE frequency.

The ability of the theory to model the evolution of the frequency of ACs is a powerful diagnostic tool of MHD spectroscopy to determine the precise time evolution of $q_{\text {min }}$ during the cascades. When the motional Stark effect diagnostic becomes operational, it will be possible to calibrate its $q$ profile measurements at the time of the ACs. The time of the appearance of the ACs has been used in JET to know when to heat strongly to produce 
internal transport barriers (ITBs), but up to now no clear ITBs have been found in the current rise associated with ACs in C-Mod. With the addition of lower hybrid current drive, it may be possible to control the ACs and drive ITBs in the current rise for advanced tokamak operation.

\section{V.C. Current Rise High-Frequency Modes Above the TAE Gap}

In addition to AEs with frequencies below the TAE gap, there are also eigenmodes in the current rise with twice the $\mathrm{AC}$ frequencies and twice the TAE frequencies. Figure 13 shows a comparison of a PCI signal with a magnetic pickup coil signal where there is a clear secondharmonic sideband of the dominant AC from about 0.14 to $0.18 \mathrm{~s}$ at exactly twice the frequency (within $\pm 3 \mathrm{kHz}$ ) and twice the slope of the dominant AC in the PCI signal. Its amplitude is much smaller than that of the first harmonic, indicating the nonlinear character of these modes as expected by theory. ${ }^{28,42}$ Note that this is not an instrumental effect in that the second harmonic is also faintly visible in the magnetic pickup coil signals, indicating that it is either a strong toroidal mode coupling or a strong fast particle drive that enhances the nonlinearity to make the second harmonic visible in the PCI data. Through a detailed examination of the data, before the frequency peaks the second harmonic is faintly visible on the magnetic pickup coil signals, suggesting that this mode is more core localized or has shorter wavelength and falls off more rapidly with radius than the first harmonic. Just after the frequency peaks and rolls over, the second harmonic becomes more clearly visible on the magnetic coils, indicating a transition to an eigenmode with an extended radial structure.
Figure 14 shows the time-averaged Fourier transform of both a core PCI signal and an edge magnetic coil signal during the rise of an $\mathrm{AC}$. The amplitude ratio of the first to the second harmonic at this time on the PCI is $\sim 36$, while the ratio on the pickup coil is $\sim 8$. The signalto-noise ratio on the PCI is larger for the second harmonic and about the same for the first harmonic compared to the magnetic signal, indicating that the low- $n$ (probably $n=1$ ) first harmonic has a broader radial structure out to the plasma edge than the more core-localized second harmonic. Note, however, that the PCI signal is uncalibrated, so there could be a frequency dependence of the amplitude that is not taken into account.

Downward-sweeping high-frequency modes beginning at about $1 \mathrm{MHz}$ are also observed, which end just before the appearance of the ACs (Fig. 15). The frequency of this mode agrees well with the calculated center of the gap EAE frequency ${ }^{8}$ at $q=2\left(\omega_{\mathrm{EAE}}=v_{A} / q R\right)$. The calculated center of the gap TAE frequency for $q=2$ also agrees well with the TAEs at the top of the AC from 0.17 to $0.2 \mathrm{~s}$ in Fig. 15. These data suggest that the EAE appears as the $q=2$ surface enters the plasma in the current rise so that these modes also provide an accurate diagnostic constraint on the $q$ profile.

\section{FAST-PARTICLE CALCULATIONS}

The TRANSP/FPPRF code ${ }^{46}$ was used with TORIC (Ref. 47) to calculate the rf electric field distribution to estimate the fast-particle tail temperature profile. Figure 16 shows the calculated time evolution of the fast hydrogen ion tail temperature, together with its radial profile and the rf power deposition during the TAEs of

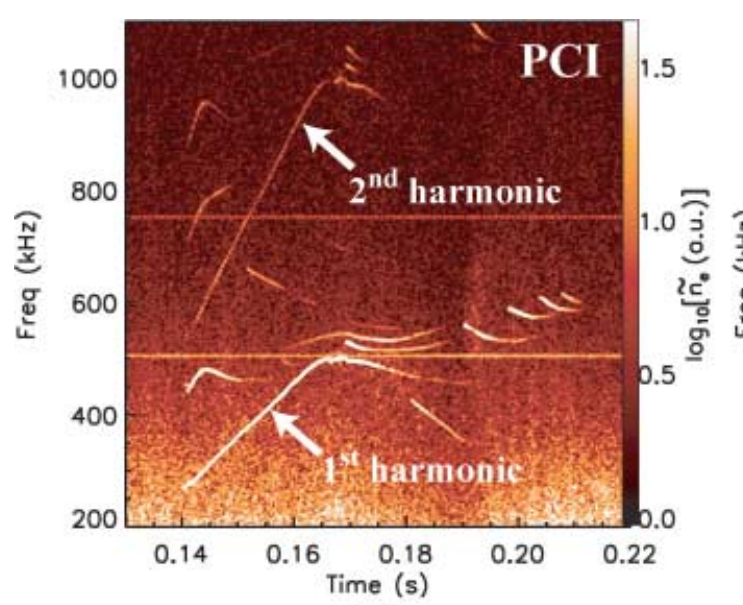

(a)

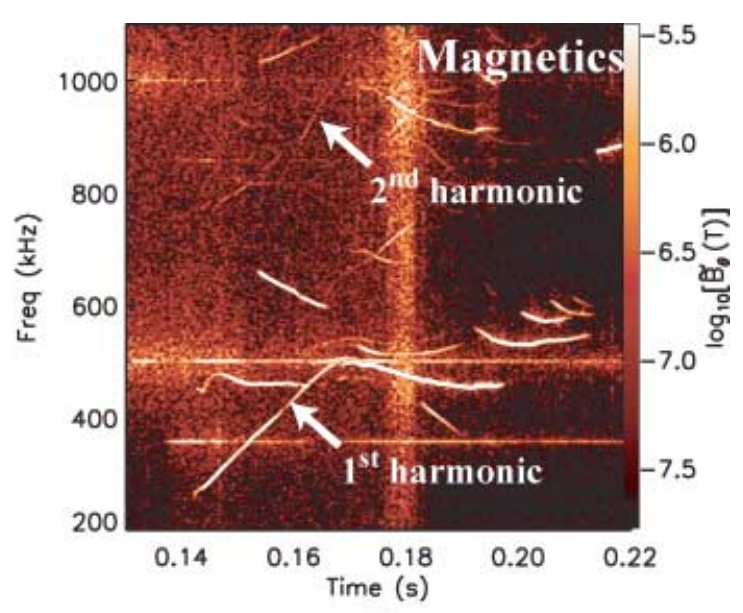

(b)

Fig. 13. (a) Fourier spectrogram of PCI density fluctuations during the current rise, showing a clear second harmonic of the dominant AC. (b) Fourier spectrogram of a poloidal field pickup coil signal, showing that the second harmonic is only very faintly visible, somewhat more clearly as the frequency peaks and rolls over into a TAE. 

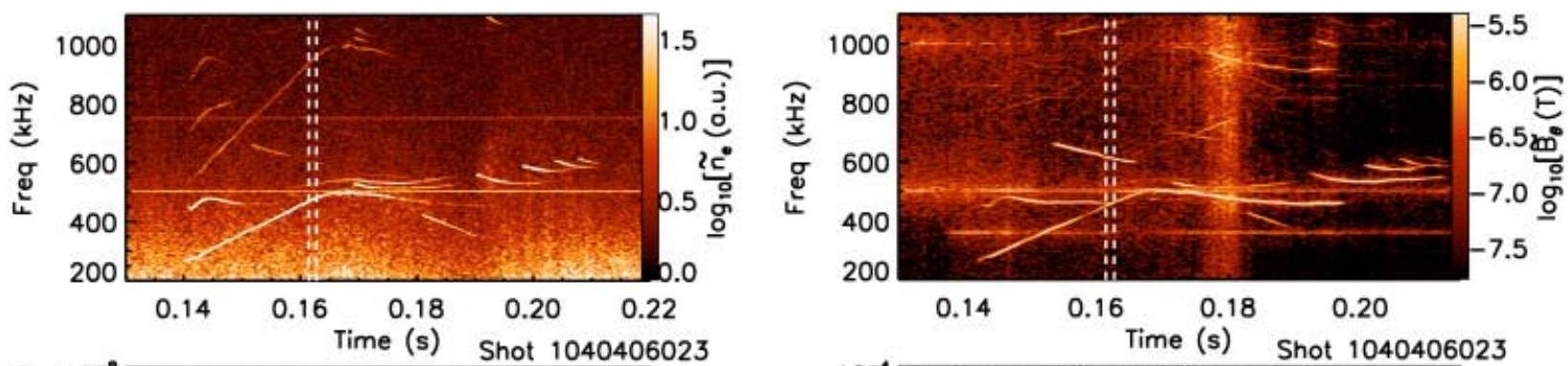

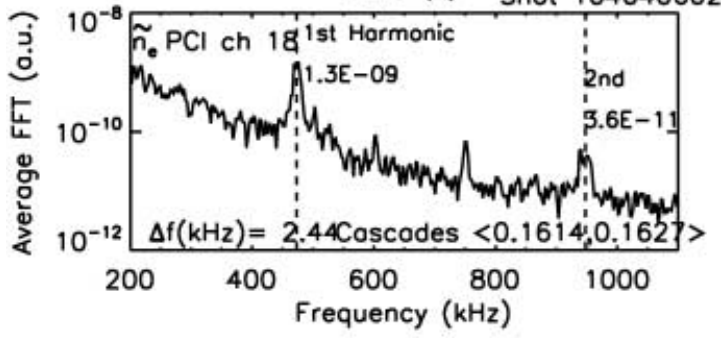

(a)

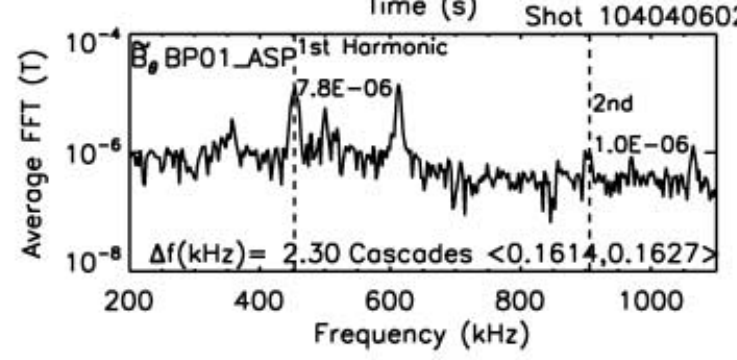

(b)

Fig. 14. (a) Fourier spectrogram of a core PCI signal, together with its time average from 0.1614 to $0.1627 \mathrm{~s}$ during the rise of an $\mathrm{AC}$ and its second harmonic. (b) Fourier spectrogram of an edge magnetic pickup coil signal and its time average over the same time interval.

Fig. 11. The fast-ion tail temperature in TRANSP is defined as $T_{H}=2 / 3\langle E\rangle$, where $\langle E\rangle$ is the flux surfaceaveraged mean energy of the hydrogen minority distribution in the lab frame. For the single AC found in earlier

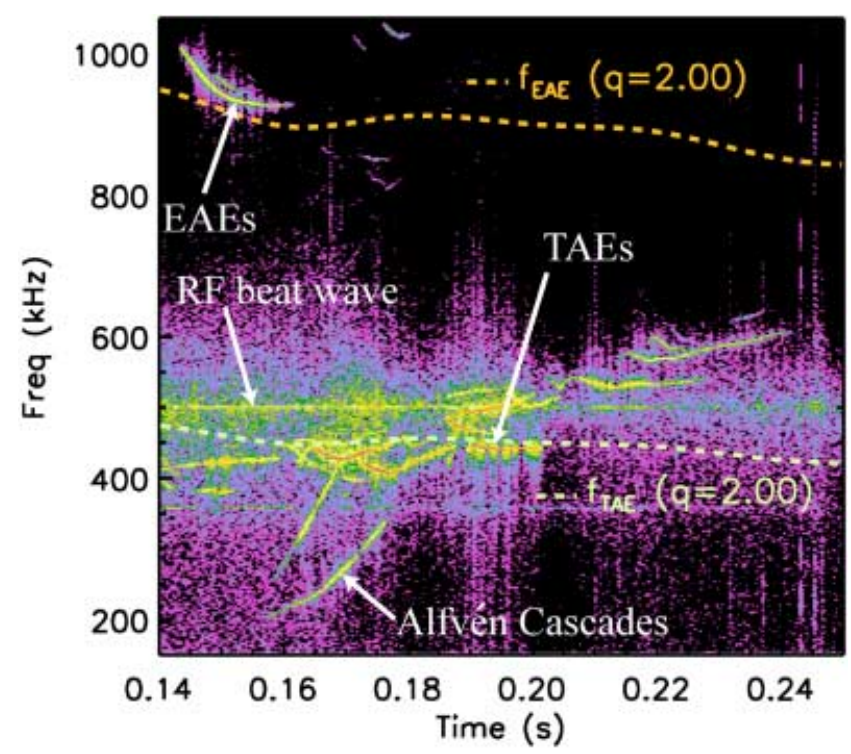

Fig. 15. Fourier spectrogram of a magnetic pickup coil signal showing a downward sweeping mode at $1 \mathrm{MHz}$ that agrees with the calculated center of the gap EAE frequency $\left(\omega_{\mathrm{EAE}}=v_{A} / q R\right)$ for $q=2$. ACs appear just after the EAE ends, and the frequency of the TAEs at the top of the cascades also agrees well with the calculated center of the gap TAE frequency for $q=2$. experiments, shown in Fig. 10, the peak fast $\mathrm{H}$ ion energy was found to be only $150 \mathrm{keV}$ due to substantially lower ICRF input power.

Under the high-input power and relatively lowdensity conditions of Fig. 11, with $\bar{n}_{e}=1.7 \times 10^{20} \mathrm{~m}^{-3}$, the calculated fast-ion tail temperature can exceed 300 $\mathrm{keV}$, with peaked $\mathrm{rf}$ power densities of $75 \mathrm{~W} / \mathrm{cm}^{-3}$. For comparison, the Stix effective temperature ${ }^{48}$ for these conditions $\left(T_{e}=3200 \mathrm{eV}, n_{H} / n_{e} \approx 0.04\right)$ reaches almost $1 \mathrm{MeV}$. So, for these high power densities, $300 \mathrm{keV}$ may be a reasonable estimate of the fast-ion tail temperature, but there are large uncertainties in the tail temperature because of the uncertainty in the core fast-ion concentration, which is based on a spectroscopic measurement of the ratio $\mathrm{H}_{\alpha} / \mathrm{D}_{\alpha}$ in the plasma edge. Similar results were obtained for the sawtooth stabilization case shown
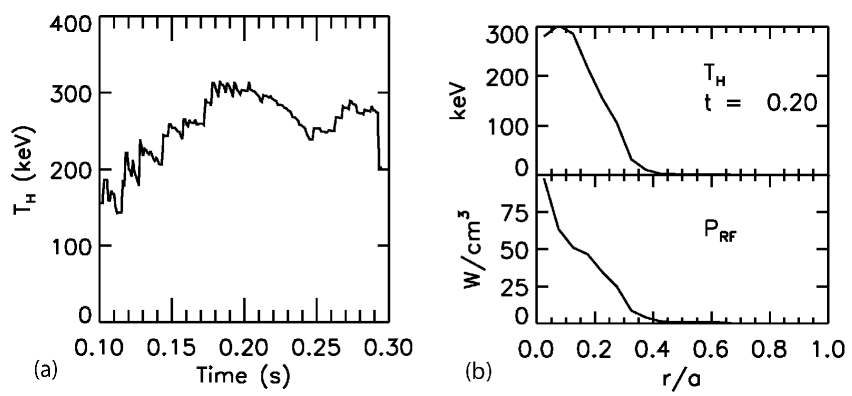

Fig. 16. (a) TRANSP/FPPRF calculated fast hydrogen ion tail temperature as a function of time during the AEs of Fig. 11. (b) Radial profiles of the fast-ion tail temperature and the $\mathrm{rf}$ power deposition at $0.2 \mathrm{~s}$. 
in Fig. 4 with fast-ion tail temperatures exceeding $300 \mathrm{keV}$ during the modes.

These high fast-ion tail temperatures correspond to fast-ion velocities that reach $v_{H} / v_{A} \approx 0.8$, which is more than sufficient to excite TAEs for a precession drift resonance. ${ }^{49}$ The very high power densities present in these ICRF-heated plasmas provide sufficient power per particle even at these densities to drive such relatively fast ion velocities, and the AEs themselves are valuable as a qualitative measure of the time evolution and substantial energy of the fast-ion distribution. More detailed measurements of the fast-ion distribution to compare with $\mathrm{AE}$ activity await results from the compact neutral particle analyzer.

\section{CONCLUSIONS}

Stable and unstable AEs are measured on Alcator C-Mod with equilibrated ion and electron temperatures at the same densities and toroidal fields and in the same toroidal mode range as expected in ITER. Moderate- $n$, from 4 to 14, stable TAE resonances have been excited, and initial scaling of their damping rates has been measured. The measured damping rate for this range of moderate- $n$ stable TAEs does not show any clear dependence on the edge magnetic shear, in contrast to the increasing damping rates with increasing shear found on JET for low $n \leq 2$, modes with monotonic $q$ profiles. ${ }^{14}$ While these results may indicate that the damping of low- $n$ modes is dominated by the edge and that of moderate- $n$ modes is not, the results could be masked by a change in damping due to changing $n$ numbers during the scan. More shape scan experiments measuring the TAE damping rates on C-Mod and, with new moderate- $n$ antennas, on JET are required to better define the damping rate dependence on shape and toroidal mode number for moderate- $n$ modes.

During sawtooth stabilization with ICRF, multiple- $n$ fast particle-driven modes coalesce to a single frequency and mode just before the sawtooth collapse. Nova-K modeling finds only stable modes under these conditions, perhaps indicating that they are energetic particle-driven modes that are not modeled by the code.

ACs during the current rise with strong ICRF heating have been used to constrain the $q$ profile, indicating a very flat shear profile across the plasma core. A second harmonic sideband of the ACs is sometimes observed on PCI measurements in the core of the plasma with oneeighth the amplitude of the first harmonic, indicating the nonlinear nature of these modes, as expected by theory. Downward sweeping modes around $1 \mathrm{MHz}$ appear just before the ACs whose frequency agrees with the EAE frequency for $q=2$, indicating that the $q=2$ surface appears in the plasma just before the cascades.

TRANSP calculations indicate that the fast hydrogen ion tail temperatures reach $300 \mathrm{keV}$ with peaked ICRF power densities up to $75 \mathrm{~W} / \mathrm{cm}^{3}$ even with densities up to $1.7 \times 10^{20} \mathrm{~m}^{-3}$. This high power per particle drives fastion velocities up to $v_{H} / v_{A} \approx 0.8$, which is more than sufficient to excite TAEs under these conditions according to theory. The presence of the AEs is also a valuable diagnostic of the time evolution of the fast ions.

\section{ACKNOWLEDGMENTS}

We would like to thank the Alcator C-Mod team members for all their hard work keeping the tokamak and its diagnostics running to acquire the data used in these experiments. This work is supported by the U.S. Department of Energy contract DE-FC02-99ER54512.

\section{REFERENCES}

1. R. AYMAR et al., Nucl. Fusion, 41, 1301 (2001).

2. J. CANDY and M. N. ROSENBLUTH, Nucl. Fusion, 35, 1069 (1995).

3. N. N. GORELENKOV, C. Z. CHENG, and W. M. TANG, Phys. Plasmas, 5, 3389 (1998).

4. N. N. GORELENKOV et al., Nucl. Fusion, 43, 594 (2003).

5. K.-L. WONG, Plasma Phys. Control. Fusion, 41, R1 (1999).

6. I. H. HUTCHINSON et al., Phys. Plasmas, 1, 1511 (1994).

7. M. N. ROSENBLUTH and P. H. RUTHERFORD, Phys. Rev. Lett., 34, 1428 (1975).

8. C. Z. CHENG, L. CHEN, and M. S. CHANCE, Ann. Phys (N.Y.), 161, 21 (1985).

9. G. Y. FU and J. W. VAN DAM, Phys. Fluids B, 1, 1949 (1989).

10. R. BETTI and J. P. FREIDBERG, Phys. Fluids B, 4, 1465 (1992).

11. F. ZONCA and L. CHEN, Phys. Fluids B, 5, 3668 (1993).

12. G. Y. FU et al., Phys. Plasmas, 3, 4036 (1996).

13. A. FASOLI et al., Phys. Rev. Lett., 75, 645 (1995).

14. D. TESTA and A. FASOLI, Nucl. Fusion, 41, 809 (2001).

15. A. FASOLI et al., Plasma Phys. Control. Fusion, 44, B159 (2002).

16. C. E. KIERAS and J. A. TATARONIS, J. Plasma Phys., 28, 395 (1982).

17. J. A. SNIPES, D. SCHMITTDIEL, A. FASOLI, R. S. GRANETZ, and R. R. PARKER, Plasma Phys. Control. Fusion, 46, 611 (2004).

18. J. A. SNIPES et al., Phys Plasmas, 12, 056102 (2005).

19. M. SAIGUSA et al., Plasma Phys. Control. Fusion, 40, 1647 (1998).

20. L.-G. ERIKSSON et al., Phys. Rev. Lett., 81, 1231 (1998).

21. W. W. HEIDBRINK et al., Nucl. Fusion, 39, 1369 (1999).

22. S. BERNABEI et al., Phys. Rev. Lett., 84, 1212 (2000).

23. F. PORCELLI, Plasma Phys. Control. Fusion, 33, 1601 (1991).

24. H. KIMURA et al., Nucl. Fusion, 38, 1303 (1998).

25. S. E. SHARAPOV et al., Phys. Plasmas, 9, 2027 (2002).

26. J. A. SNIPES et al., Plasma Phys. Control. Fusion, 42, 381 (2000).

27. R. NAZIKIAN et al., Phys. Rev. Lett., 91, 125003 (2003). 
28. H. L. BERK, D. N. BORBA, B. N. BREIZMAN, S. D. PINCHES, and S. E. SHARAPOV, Phys. Rev. Lett., 87, 185002 (2001).

29. A. B. MIKHAIlOVSKII, G. T. A. HUYSMANS, W. O. K. KERNER, and S. E. SHARAPOV, Plasma Phys. Rep., 23, 844 (1997).

30. A. MAZURENKO et al., Phys. Rev. Lett., 89, 225004 (2002).

31. N. P. BASSE et al., Fusion Sci. Technol., 51, 476 (2007).

32. A. FASOLI et al., Nucl. Fusion, 35, 1485 (1995).

33. W. BURKE et al., "Broadband Amplifiers for the Active MHD Diagnostic on Alcator C-Mod," Proc. 20th IEEE/NPSS Symp. Fusion Engineering (SOFE), p. 363, IEEE (2003).

34. J. A. SNIPES et al., Proc 26th EPS Conf., Maastricht, The Netherlands, Vol. 23J, p. 293, European Physical Society (1999).

35. M. BRAMBILLA, Kinetic Theory of Plasma Waves, p. 319, Clarendon Press, Oxford, England (1998).

36. R. F. HEETER et al., presented at Fifth IAEA Technical Committee Mtg. Alpha Particles in Fusion Research (unpublished).

37. W. KERNER et al., Nucl. Fusion, 38, 1315 (1998).

38. I. H. HUTCHINSON et al., Plasma Phys. Control. Fusion, 41, A609 (1999).
39. S. D. PINCHES et al., Proc. 24th European Conf. Controlled Fusion and Plasma Physics, Berchtesgaden, Germany, Vol. 21A, Part IV, p. 1557 (1997).

40. G. J. KRAMER, S. E. SHARAPOV, R. NAZIKIAN, N. N. GORELENKOV, and R. V. BUDNY, Phys. Rev. Lett., 92, 015001 (2004).

41. G. Y. FU and C. Z. CHENG, Phys. Fluids B, 4, 3722 (1992).

42. F. ZONCA et al., Phys. Plasmas, 9, 4939 (2002).

43. G. J. KRAMER et al., Phys. Rev. Lett., 83, 2961 (1999).

44. S. E. SHARAPOV et al., Phys. Rev. Lett., 93, 165001 (2004).

45. A. MAZURENKO, "Phase Contrast Imaging on the Alcator C-Mod Tokamak," PhD Thesis, Massachusetts Institute of Technology, Cambridge, Massachusetts (2001).

46. G. W. HAMMETT, "Fast Ion Studies of Ion Cyclotron Heating in the PLT Tokamak," PhD Thesis, Princeton University, Princeton, New Jersey (1986).

47. M. BRAMBILlA, Plasma Phys. Control. Fusion, 41, 1 (1999).

48. T. H. STIX, Nucl. Fusion, 15, 737 (1975).

49. H. BIGLARI, F. ZONCA, and L. CHEN, Phys. Fluids B, 4, 2385 (1992). 